\title{
Management of acute exacerbations of airways disease: advice for the non-respiratory physician
}

\author{
Authors: Lynn Elsey ${ }^{\mathrm{A}}$ and David Allen ${ }^{\mathrm{B}}$
}

\begin{abstract}
Airways disease encompasses a number of conditions including asthma and chronic obstructive pulmonary disorder (COPD). In this article we describe the management of patients with acute exacerbations of airways disease.
\end{abstract}

\section{Introduction}

Asthma is a condition characterised by reversible obstruction of the airways due to airway inflammation that results in bronchial smooth muscle constriction, endobronchial mucosal oedema and mucus hypersecretion and is associated with bronchial hyperresponsiveness. Patients may suffer from episodes of intermittent worsening. Asthma attacks may occur at random with no obvious precipitating factors or may be caused by poor adherence to treatment or by triggers such as exposure to inhaled allergens (eg house dust mites, animal fur or pollen), tobacco smoke, air pollution, cold air, exercise, occupational factors or viruses such as rhinovirus and influenza.

By contrast, chronic obstructive pulmonary disease (COPD) is characterised by persistent, often fixed, airway obstruction that results in persistent respiratory symptoms, especially breathlessness. COPD is an umbrella term that includes emphysema and chronic bronchitis. It is most commonly associated with a history of exposure to tobacco smoke but can also occur in non-smokers. ${ }^{1}$ Exacerbations are acute episodes of worsening COPD symptoms (such as increased breathlessness, cough and sputum) that are beyond normal dayto-day variations. Airway inflammation in COPD is predominantly neutrophil-driven, although it is now known that some patients with COPD and bronchiectasis have an 'asthmatic' component with steroidresponsive eosinophil-driven airway inflammation and a degree of bronchodilator responsiveness. ${ }^{2}$ A peripheral blood eosinophil count of $>0.3 \times 10^{9} / \mathrm{L}$ can identify patients who may have steroid-responsive airway inflammation. ${ }^{3}$ It is also recognised that some patients with asthma can develop persistent airflow obstruction. ${ }^{4}$

\section{Clinical assessment}

The aim of clinical assessment is to confirm the diagnosis of either asthma or COPD, assess the likely trigger(s) for the exacerbation and assess the severity of the exacerbation to guide initial treatment.

Authors: ${ }^{\text {A }}$ lead respiratory pharmacist, Manchester University NHS Foundation Trust, Manchester, UK; ${ }^{B}$ consultant respiratory physician, Manchester University NHS Foundation Trust, Manchester, UK

\section{Key points}

\section{Asthma}

Monitor peak expiratory flow rate (PEFR) throughout admission and consider intravenous magnesium if PEFR $<50 \%$ of predicted or of patient's known recent best.

Antibiotic therapy should only be considered if patients have clinical signs of infection, such as raised C-reactive protein and white cell count.

Optimise inhaler technique.

Check inhaler adherence and escalate/de-escalate treatment as appropriate. Ensure any treatment changes are formally reviewed within 8 weeks.

If the patient is adherent to optimal inhaled therapy and has required more than two courses of prednisolone in the last 12 months, refer to specialist services.

\section{Chronic obstructive pulmonary disorder}

Aim for oxygen saturations of $88-92 \%$ with controlled $\mathrm{O}_{2}$ therapy at admission if there is a history of type 2 respiratory failure.

Obtain an accurate assessment of oxygen saturation, partial pressure of $\mathrm{CO}_{2}$ and $\mathrm{pH}$ through blood gas analysis.

Consider antibiotics only if there are clinical signs of and objective evidence for infection.

If the patient has had repeated courses of oral steroids, remember to wean them slowly off steroids and not to stop them abruptly.

Optimise inhaler technique.

Review inhaled therapy against local/national guidelines; if the peripheral blood eosinophil count isn't raised, consider stopping inhaled corticosteroids and switching to a long-acting beta agonist / long-acting muscarinic antagonist inhaler.

KEYWORDS: asthma, COPD, airways disease

DOI: $10.7861 /$ clinmed.2021-0649 
Table 1. Clinical features differentiating chronic obstructive pulmonary disorder and asthma

\begin{tabular}{lll} 
& $\begin{array}{l}\text { Chronic obstructive } \\
\text { pulmonary disorder }\end{array}$ & Asthma \\
$\begin{array}{l}\text { Smoker or ex-smoker } \\
\text { Symptoms under } \\
\text { the age of } 35 \text { years } \\
\begin{array}{l}\text { Chronic productive } \\
\text { cough }\end{array}\end{array}$ & Rare & Possibly \\
$\begin{array}{l}\text { Breathlessness } \\
\text { Often }\end{array}$ & Persistent and & Uncommon \\
$\begin{array}{l}\text { Night-time waking } \\
\text { with breathlessness } \\
\text { and/or wheeze }\end{array}$ & Uncommon & Short time periods \\
$\begin{array}{l}\text { Significant diurnal } \\
\text { or day-to-day } \\
\text { variability of } \\
\text { symptoms }\end{array}$ & Uncommon & Common \\
\hline
\end{tabular}

\section{History}

Many patients presenting with an exacerbation of airway disease will already have a pre-existing diagnosis of either asthma or COPD. Sometimes an acute exacerbation can be an initial presentation of either condition (see Table 1 for clinical features differentiating asthma from COPD). ${ }^{5}$ A thorough drug history should be undertaken. Consider adherence to preventer inhalers, which can lead to poor asthma control, history of beta blocker and NSAID use as these can exacerbate asthma attacks.

\section{Examination}

This is the key step in identifying the severity of the exacerbation. Evidence of altered consciousness level in either asthma or COPD should prompt urgent assessment of the arterial blood gases to identify signs of type 2 respiratory failure, which may require prompt treatment and assessment by critical care. A normal partial pressure of $\mathrm{CO}_{2}\left(\mathrm{PaCO}_{2}\right)$ in a patient with a severe asthma attack can be a sign of impending respiratory fatigue. It is recommended that repeated assessment and measurement of arterial blood gases is performed in patients presenting with acute severe asthma not responding to initial therapy.

Assessment of the work of breathing, the use of accessory muscles and the respiratory rate can also guide the determination of severity and impending respiratory muscle fatigue that could lead to type 2 respiratory failure.

Clinical examination should confirm the presence of bilateral breath sounds, since spontaneous pneumothorax can occur in both patients with asthma and COPD. The typical wheeze in asthma is expiratory and polyphonic due to wide variations in airway calibre; however, in life-threatening asthma the chest can be silent with soft breath sounds, a sign of the need for urgent review by the critical care team. Monophonic or unilateral wheeze can be a sign of large airway obstruction, such as may be caused by tumour. Clinical examination may also reveal bronchial breathing and/or localised crackles associated with pneumonia. Squeaks should not be confused with wheezes. They usually occur in mid to late inspiration and usually indicate interstitial lung diseases such as hypersensitivity pneumonitis and pulmonary fibrosis, although they may also be heard in obliterative bronchiolitis. Fine crackles are often heard in patients with interstitial lung disease and are also found in patients with pulmonary oedema due to left ventricular failure.

Patients with COPD should be assessed for the presence of signs of cor pulmonale (raised jugular venous pressure and peripheral oedema) that may worsen during an exacerbation as well as for the presence of other comorbidities such as ischaemic heart disease, thromboembolic disease and lung cancer.

Peak expiratory flow rate (PEFR) is used in asthma to aid the assessment of severity and to monitor for response to therapy but has little role in assessing patients with COPD. Measurement of arterial oxygen saturation by pulse oximetry $\left(\mathrm{SpO}_{2}\right)$ is routinely employed in both asthma and COPD and used to titrate oxygen therapy, and arterial blood gas measurements are used in both conditions to assess acid-base balance and $\mathrm{PaCO}_{2}$. There is no evidence to support routine use of spirometry in the acutely unwell asthmatic patient.

\section{Management}

\section{Management of acute asthma}

Acute exacerbations of asthma can be classified as moderate, severe or life-threatening (see Table 2). ${ }^{6}$

\section{Table 2. Levels of severity of acute asthma attacks} in adults ${ }^{6}$

Moderate acute Increasing symptoms

asthma $\quad>$ PEFR $>50-75 \%$ best or predicted

$>$ No features of acute severe asthma

Severe acute Any one of the following:

asthma > PEFR $33 \%-50 \%$ best or predicted

$>$ Respiratory rate $\geq 25$ breaths $/ \mathrm{min}$

$>$ Heart rate $\geq 110$ beats/min

$>$ Inability to complete sentences in one breath

Life-threatening Any one of the following in a patient with asthma severe asthma:

\section{Clinical signs \\ $>$ Altered conscious level \\ $>$ Exhaustion \\ $>$ Arrhythmia \\ $>$ Hypotension \\ $>$ Cyanosis \\ $>$ Silent chest \\ $>$ Poor respiratory effort}

Measurements
$>\mathrm{PEFR}<33 \%$ best or predicted
$>\mathrm{SpO}_{2}<92 \%$ on air
$>\mathrm{PaO}_{2}<8 \mathrm{kPa}$ on air
$>$ 'Normal' $\mathrm{PaCO}_{2}(4.6-6.0 \mathrm{kPa})$

Near-fatal Raised $\mathrm{PaCO}_{2}$ and/or requiring mechanical asthma ventilation with raised inflation pressures

$\mathrm{PaCO}_{2}=$ partial arterial pressure of carbon dioxide; $\mathrm{PaO}_{2}=$ partial arterial pressure of oxygen; $\mathrm{PEFR}=$ peak expiratory flow rate; $\mathrm{SpO}_{2}=$ oxygen saturation measured by a pulse oximeter. 
> Give supplementary oxygen to all hypoxaemic patients with acute severe asthma, titrated to maintain an $\mathrm{SpO}_{2}$ level of $94 \%-98 \%$.

$>$ Do not delay oxygen administration in the absence of pulse oximetry but commence monitoring of $\mathrm{SpO}_{2}$ as soon as it becomes available.

$>$ Use frequent high-dose nebulised beta 2 agonists as first-line agents in patients with acute asthma and administer as early as possible. Nebulisers should be oxygen-driven. Regular nebulised therapy and the increased work of breathing can lead to a lactic acidosis; this is usually short lived and does not require specific intervention. Patients presenting with significant lactic acidosis should be investigated for possible sepsis.

> Give steroids in adequate doses $(40-50 \mathrm{mg}$ ) to all patients with an acute asthma attack; when oral administration is not possible consider regular intravenous hydrocortisone.

> Consider giving a single dose of intravenous magnesium sulphate (1.2-2 $\mathrm{g}$ by intravenous infusion over 20 minutes) to patients with acute severe asthma (PEFR $<50 \%$ of best or predicted) who have not had a good initial response to inhaled bronchodilator therapy.

$>$ Reserve intravenous beta 2 agonists or intravenous aminophylline for those patients with acute severe asthma who are not responding to initial nebulised therapy or intravenous magnesium. Patients who are not responding to the above measures and who may require intravenous bronchodilators should be assessed by critical care as early as possible.

\section{Management of acute exacerbations of COPD}

Eighty per cent of COPD exacerbations can be managed in an outpatient setting. ${ }^{3}$ In all patients presenting to hospital with an acute exacerbation the following points should be considered. ${ }^{5}$

> Short-acting beta 2 agonist bronchodilators (SABAs), with or without short-acting muscarinic antagonists (SAMAs), are recommended as initial treatment for an acute exacerbation. Review the doses that the patient is taking on admission and increase as required. If the patient is on a long-acting muscarinic antagonist (LAMA), this should be suspended while they are being treated with a SAMA.

> Inhaled bronchodilators should be delivered either by metered dose inhaler administered using a spacer device or by nebuliser driven by air to avoid worsening potential hypercapnia. Oxygen should be delivered separately from the nebuliser. The nebuliser device and air driver should be stated on all prescriptions.

$>$ Administer supplemental oxygen, aiming for oxygen saturations of $88 \%-92 \%$, while awaiting arterial blood gas results. Patients with evidence of chronic type 2 respiratory failure should then continue to receive oxygen titrated to maintaining $\mathrm{SaO} 2$ $88 \%-92 \%$ without injudicious further elevation of the $\mathrm{PaCO}_{2}$.

$>$ Consider oral or systemic steroids, for example, prednisolone $0.5-0.6 \mathrm{mg} / \mathrm{kg}$ body weight for 5-10 days. If the patient has had repeated courses of oral steroids recently, they will require a subsequent tapering regimen.

$>$ Consider antibiotics for 5-7 days if there is clinical evidence of infection and/or the C-reactive protein is significantly elevated. Refer to local antimicrobial guidelines for selection of first-line therapy and always check the patient's allergy status. Consider the possibility of significant $\mathrm{QT}_{\mathrm{C}}$ prolongation for relevant antibiotics.

> Methylxanthines, such as theophylline and aminophylline, are not routinely indicated due to the side effect risk. In severe cases infusions of aminophylline may be considered but blood levels must be monitored closely.
$>$ Non-invasive ventilation should be considered if appropriate in non-responding patients, particularly when it is not possible to adequately oxygenate the patient without dangerously further elevating the $\mathrm{PaCO}_{2}$. If this is done on the respiratory or nonrespiratory ward rather than in the high-dependency unit or intensive care unit, consideration must be given to the adequate provision of trained nursing support and monitoring, including of the $\mathrm{PaCO}_{2}$.

\section{Assessment of the patient's regular treatment}

Important points to consider when reviewing the treatment of a patient admitted with an acute exacerbation of asthma or COPD include the following.

> Obtain a full drug history, including of the number of steroid and antibiotic prescriptions the patient has received in the previous year.

$>$ Check the patient's adherence to inhaled corticosteroid (ICS) or combination (ICS / long-acting beta agonist (LABA)) therapies, using general practitioner (GP) or pharmacy records. Educate the patient on the importance of regular preventer inhaler use as poor compliance is associated with poor asthma control.

> Review use of SABA inhalers in asthma: use of more than one canister each month is associated with a three-fold increased risk of attendance at emergency departments, and of hospitalisation.

> Assess the patient's inhaler technique. Poor inhaler technique is one of the commonest causes of failure of inhaled treatments. Even with excellent inhaler technique only $10 \%-20 \%$ of the delivered dose reaches the airways. Spacer devices generally improve delivery significantly provided that they are used properly. In addition, ensure the patient knows how many doses their inhaler device will deliver.

$>$ Review the patient's blood eosinophil count:

$>$ in asthma patients a raised eosinophil count may indicate ongoing inflammation despite optimal therapy (suggested by an eosinophil count of 150 cells/ $\mu \mathrm{L}$ or higher)

> in COPD, this will allow decision making about the role of inhaled steroids in their treatment plan; patients with eosinophil counts equal to or greater than 300 cells/ $\mu \mathrm{L}$ are more likely to have some inhaled steroid responsiveness.

> If the patient has received multiple courses of oral steroids recently then a gradual tapering of the acute course will be required.

$>$ Routine prescriptions of antibiotics are not indicated for acute asthma; rather, the decision to start antibiotics should be supported by objective evidence of infection.

> Any treatment changes made during admission should be highlighted to the patient's GP and the response to them should be reviewed by a healthcare professional within $4-8$ weeks. This arrangement should be put in place prior to hospital discharge.

\section{Escalating and de-escalating treatment}

Baseline treatment should only be amended if adherence to the current treatment regimen has been confirmed. The severity of disease and its response to treatment can only be properly assessed if the patient has been taking their treatment regularly. Adherence can be assessed using data from the patient's GP and pharmacy records. Good adherence to inhaled therapies is classified as $>75 \%$ of preventer inhaler prescriptions collected (9/12 preventer inhalers in a year).

Patients may also be unintentionally non-adherent due to poor inhaler technique. This should be reviewed using the seven steps of good inhaler technique. ${ }^{8}$ 
1. Prepare the inhaler device.

2. Prepare or load the dose.

3. Breathe out, fully and gently, but not into the inhaler.

4. Tilt the chin up slightly and place the inhaler mouthpiece in the mouth, sealing the lips around the mouthpiece.

5. Breathe in fully in one of the following ways:

> Aerosol, eg pressurised metered dose inhaler: slow and steady

$>$ Soft mist inhaler: slow and steady

$>$ Dry powder inhaler: quick and deep

6. Remove inhaler from the mouth and hold the breath for at least 10 seconds.

7. Wait for a few seconds, then repeat as necessary.

\section{Inhaled triple therapy in COPD}

Particularly at high dose, ICS can increase the risk of pneumonia in COPD patients and not all COPD patients will have steroid-responsive airway inflammation. As already described, blood eosinophil levels can be used to guide the prescribing of ICS in COPD.

Patients should be reviewed and stepped down to inhaled LABA/ LAMA therapy if they do not have evidence of steroid responsive airway inflammation (Box 1). ${ }^{3}$

Triple therapy, with the addition of a LAMA, may be appropriate in some asthma patients who continue to exacerbate despite taking a medium dose ( $\geq 800$ micrograms/day of beclomethasone dipropionate or equivalent) of an ICS combined with a LABA with confirmed good inhaler technique. ${ }^{6}$

\section{Referral to specialist services}

Patients should be referred to specialist asthma services if they have severe asthma requiring treatment with high dose ICS or frequent exacerbations or are at high risk of asthma death. These patients can be assessed by specialist multidisciplinary teams to review their diagnosis, optimise comorbidities and consider treatment with biologic therapies such as mepolizumab. This can avoid the need for continuous maintenance oral corticosteroids and the risk of associated long-term systemic adverse effects. ${ }^{6}$

COPD patients should be referred to specialist services if clinically indicated. This may be for a variety of reasons, including suspected severe COPD, rapid decline in $\mathrm{FEV}_{1}$, exclusion of comorbidities (such as bronchiectasis, pulmonary embolism and lung cancer), consideration for long-term oxygen therapy or for long term nebuliser therapy. ${ }^{5}$

Box 1. Factors to consider when starting inhaled corticosteroids in chronic obstructive pulmonary disorder

\section{Use strongly supported}

History of hospitalisation with $\geq 2$ moderate exacerbations per year

> Blood eosinophils 300 cells $/ \mu \mathrm{L}$

\section{Consider use}

One moderate exacerbation of chronic obstructive pulmonary disorder per year

> Blood eosinophils 100-300 cells/ $\mu \mathrm{L}$

Use not advised

Repeated pneumonias

Blood eosinophils $<100$ cells $/ \mu \mathrm{L}$

History of mycobacterial infection

\section{Future directions}

A new joint guideline from the National Institute for Health and Care Excellence, the British Thoracic Society and the Scottish Intercollegiate Guidelines Network (NICE/BTS/SIGN) for the diagnosis and management of asthma is in development. This joint guideline will provide one reference source and help to clarify the best management approach to asthma care. Asthma care has changed rapidly over the last 5 years with the introduction of monoclonal antibody biologic therapies for severe disease. There is now greater focus on identifying high-risk patients earlier to reduce their exposure to steroids and the long-term damage that they can cause. An acute admission is a good opportunity to check adherence to treatment and to optimise inhaled therapies. It is also an opportunity to highlight if a patient has had more than two courses of prednisolone and requires a referral to a severe asthma service for assessment for biologic treatment.

The future of COPD treatment will have a focus on optimising inhaled therapies. It is vital to ensure that patients are on the correct inhalers and that they know how to use them properly. Additional factors such as blood eosinophil counts should also be considered. If patients do not show evidence of eosinophilic inflammation, the appropriateness of inhaled steroids should be reviewed and substitution of a combination LABA/LAMA inhaler should be considered.

\section{References}

1 Antó JM, Vermeire P, Vestbo J, Sunyer J. Epidemiology of chronic obstructive pulmonary disease. Eur Respir ] 2001;17:982-94.

2 Suzuki M, Makita H, Konno S et al. Asthma-like features and clinical course of chronic obstructive pulmonary disease. Am J Respir Crit Care Med 2016;194:1358-65.

3 Global Initiative for Chronic Obstructive Lung Disease. Global strategy for the diagnosis, management and prevention of chronic obstructive pulmonary disease. GOLD, 2021. https://goldcopd.org/ wp-content/uploads/2020/11/GOLD-REPORT-2021-v1.1-25Nov20_ WMV.pdf [Accessed 1 August 2021].

4 Moore WC, Meyers DA, Wenzel SE et al. Identification of asthma phenotypes using cluster analysis in the severe asthma research program. Am J Respir Crit Care Med 2010;181:315-23.

5 National Institute of Health and Care Excellence. Chronic obstructive pulmonary disease in over 16s: diagnosis and management. NICE Guideline NG115. NICE, 2019. www.nice.org.uk/guidance/ ng115 [Accessed 1 August 2021].

6 British Thoracic Society and Scottish Intercollegiate Guidelines Network. British guideline on the management of asthma. BTS/ SIGN, 2020. www.brit-thoracic.org.uk/quality-improvement/ guidelines/asthma [Accessed 1 August 2021].

7 Silver HS, Blanchette CM, Kamble S et al. Quarterly assessment of short-acting beta(2)-adrenergic agonist use as a predictor of subsequent health care use for asthmatic patients in the United States. J Asthma 2010;47:660-6.

8 UK Inhaler Group. Inhaler standards and competency document. UKIG, 2019. www.ukinhalergroup.co.uk/uploads/s4vjR3GZ/ InhalerStandardsMASTER.docx2019V10final.pdf [Accessed 1 August 2021].

Address for correspondence: Lynn Elsey, Wythenshawe Hospital, Southmoor Road, Wythenshawe, Manchester M23 9LT, UK.

Email: lynn.elsey@mft.nhs.uk

Twitter: @lynnelsey 